\title{
THE OPTID DATABASE: DEEP OPTICAL IDENTIFICATIONS TO THE IRAS FAINT SOURCE SURVEY
}

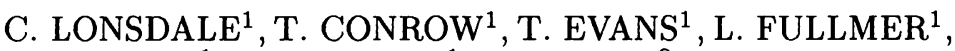 \\ M. MOSHIR ${ }^{1}$, T. CHESTER ${ }^{1}$, D. YENTIS ${ }^{2}$, \\ R. WOLSTENCROFT ${ }^{3}$, H. MacGILLIVRAY ${ }^{3}$ AND D. EGRET ${ }^{4}$ \\ ${ }^{1}$ Infrared Processing and Analysis Center/CalTech, Pasadena, \\ USA \\ ${ }^{2}$ Naval Research Laboratory, Washington DC, USA \\ ${ }^{3}$ Royal Observatory Edinburgh, Edinburgh, Scotland \\ ${ }^{4} C D S$, Strasbourg, France
}

\section{Introduction}

We use a new, robust, method to estimate the identification probabilities of optical matches from digitized plate catalogs (COSMOS/UKST Catalog of the Southern Sky Version 2, Yentis et al. 1992; The Guide Star Catalog Version 1.1, Lasker et al. 1990; The Tycho Input Catalog, Egret et al. 1992; The APM Northern Sky Catalogue, Irwin, Maddox and McMahon 1994) to sources in the IRAS Faint Source Survey (FSS; Moshir et al. 1992), including both the Catalog (FSC) and the Reject File (FSR), utilizing a new random matching procedure with the advantages that it: (1) eliminates systematic uncertainties due to many problems, such as uncertainty in $N(m)$; variations across the optical plate of magnitudes, plate limits or $N(m)$; misclassification of stars and galaxies; the assumption of Gaussian error ellipses, etc. and (2) properly calibrates the identification probabilities. We find that at high SNRs and high galactic latitudes essentially all IR star-colored sources have an optical identification with $P_{i d}>99 \%$. At high SNRs and high galactic latitudes, $\sim 90 \%$ of all IR galaxy-colored sources have an optical identification with $P_{i d}>90 \%$.

This project was undertaken to provide a star/galaxy classification for all FSS sources and an optical (blue) magnitude, allowing users to make more sophisticated searches through the FSS for sources of a given star or galaxy type, optical/IR color range, and optical magnitude range, and to 
search for unusual objects such as high redshift galaxies, brown dwarfs and IR-bright QSOs. OPTID can also be used to significantly improve IRAS positions and to help discriminate true from false low signal-to-noise IR sources. To access OPTID telnet to xcatscan.ipac.caltech.edu.

\section{Method}

We assume there is only one correct optical match to each IR source, but we do not assume that it is either the closest or the brightest. Our method can easily be generalized for the case of multiple true matches (e.g., an interacting galaxy pair). We allow the possibility that the match is fainter than the optical catalog limit.

For the $i t h$ candidate optical identification within the search area (chosen to be $4 \sigma$ to optimize trade-offs between completeness and reliability): the Likelihood Ratio is $L R_{i}=\frac{Q e^{-r_{i}^{2} / 2}}{2 \pi \sigma_{\operatorname{maj}} \sigma_{\min } N\left(<m_{i}\right)}$, where $Q$ is the probability true identification exists in the optical catalog, $r$ is the source separation, $\sigma$ is the positional uncertainty ellipse, and $N\left(<m_{i}\right)$ gives the integral background counts brighter than magnitude $m_{i}$. At each position we calculate LR for each candidate optical match to each IR source, but use it only as a relative weight for each match, because its absolute value is very sensitive to match magnitude. The Reliability of each association with a given LR is defined as the number of true associations divided by the number of true plus number of random associations for a given subclass of sources (binned by star/galaxy type, galactic latitude and SNR): $R_{i}\left(L R_{i}\right)=\frac{N_{\text {true }}\left(L R_{i}\right)}{N_{\text {true }}\left(L R_{i}\right)+N_{\text {false }}\left(L R_{i}\right)}$. Finally, we compute the Identification Probability $P_{i d}$ for each match by taking into account the number of matches found for each source and their individual Reliabilities, including the possibility that no match exists on the optical plate: $P_{i d_{i}}=\frac{Q R_{i} \Pi_{j=1}^{n}\left[\left(1-R_{j}\right)\right] /\left(1-R_{i}\right)}{S}$, and $P_{n o-i d}=\frac{(1-Q) \Pi_{i=1}^{n}\left(1-R_{i}\right)}{S}$, where $S=\sum_{i=1}^{n}\left[\operatorname{num}\left(P_{i d_{i}}\right)\right]+\underset{n u m}{\operatorname{nu}}\left(P_{n o-i d}\right)$.

\section{References}

Egret, D., Didelon, P., McLean, B.J., Russell, J.L., and Turon, C. 1992, Astron. Astrophys., 258, 217.

Irwin, M., Maddox., S. and McMahon, R.G. 1994. RGO Newsletter.

Lasker, B.M., Sturch, C.R., McLean, B.J., Russell, J.L., Jenkner, H., and Shara, M.M. 1990. Astron. J., 99, 2019.

Moshir, M., et al. 1992. Explanatory Supplement to the IRAS Faint Source Survey, Version 2 JPL D-10015 8/92 (Pasadena: JPL)

Yentis, D.J., Cruddace, R.G., Gursky, H., Stuart, B.V., Wallin, J.F., MacGillivray, H.T., and Collins, C.A. 1992. in proceedings of "Digitized Optical Sky Surveys," ed. H.T. MacGillivray and E.B. Thomson; Kluwer Academic Publishers 1992, page 67 